\title{
A Rapid Route to Core Nitrogen Containing Polycyclic Aromatics
}

\section{Gategory}

Synthesis of

Materials and

Unnatural Products

\section{Key words}

cycloaddition

planarization

extended aromatics

SV/Dal

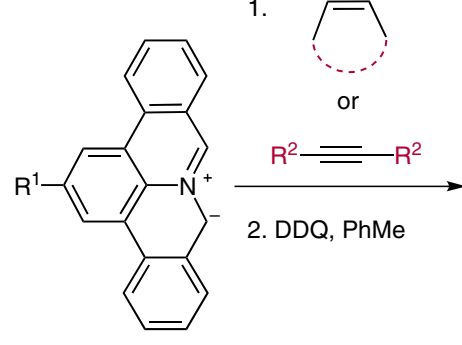

azomethine ylide

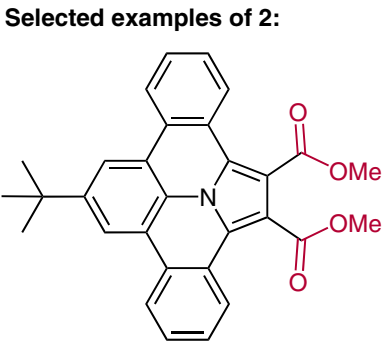

$43 \%$ yield ( 2 steps)

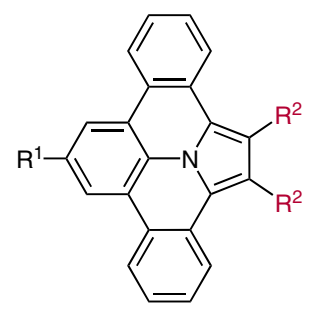

Significance: The authors report a facile method to generate novel nitrogen-containing polycyclic aromatic hydrocarbons (N-PAHs) using a metalfree approach. The sequence involves a 1,3-dipolar cycloaddition of an azomethine ylide with a dipolarophile, followed by an oxidation/planarization step; it was shown that this can be efficiently performed in one pot.
Comment: The products formed are fully planar $\pi$-extended N-PAHs that have rich photophysical properties. Installing nitrogen atoms in the core of these $\pi$-extended structures has traditionally been more synthetically challenging than decorating their periphery. Accordingly, the modular nature and rapid construction of these new extended aromatic systems should enable further research into their potential applications in nanoelectronics and related fields.

SYNFACTS Contributors: Timothy M. Swager, Myles B. Herbert 